INPLASY

PROTOCOL

To cite: Li et al. Intraoperative radiotherapy is not a better alternative to whole breast radiotherapy as a therapeutic option for early stage breast cancer: a pooling analysis of randomized controlled trials. Inplasy protocol 2020120008. doi:

10.37766/inplasy2020.12.0008

Received: 02 December 2020

Published: 02 December 2020

Corresponding author:

Min Sun

sunmin-0715@163.com

Author Affiliation:

Department of General

Surgery, Taihe Hospital, Hubei University of Medicine, Shiyan 442000, China

Support: NSFC (81902498).

Review Stage at time of this submission: Data analysis.

Conflicts of interest:

None.

\section{Intraoperative radiotherapy is not a better alternative to whole breast radiotherapy as a therapeutic option for early stage breast cancer: a pooling analysis of randomized controlled trials}

$\mathrm{Li}, \mathrm{T}^{1} ;$ Sun, $\mathrm{M}^{2}$.

Review question / Objective: Intraoperative radiotherapy (IORT) in early stage breast cancer has been studied over the years. However, it has not been established if IORT is more suitable as a therapeutic option for early stage breast cancer than whole breast radiotherapy (WBRT). Therefore, we performed a meta-analysis to compare the efficacy and safety of IORT to those of WBRT as therapeutic options for early stage breast cancer patients receiving breast-conserving surgery.

INPLASY registration number: This protocol was registered with the International Platform of Registered Systematic Review and Meta-Analysis Protocols (INPLASY) on 02 December 2020 and was last updated on 02 December 2020 (registration number INPLASY2020120008).

\section{INTRODUCTION}

Review question / Objective: Intraoperative radiotherapy (IORT) in early stage breast cancer has been studied over the years. However, it has not been established if IORT is more suitable as a therapeutic option for early stage breast cancer than whole breast radiotherapy (WBRT). Therefore, we performed a meta-analysis to compare the efficacy and safety of IORT to those of WBRT as therapeutic options for early stage breast cancer patients receiving breast-conserving surgery. 
Rationale: Radiotherapeutic approaches have been developed to overcome the problems associated with WBRT. Among them, accelerated partial breast irradiation (APBI) is gradually becoming a surrogate to WBRT, because it can effectively shorten treatment time to 1-2 weeks, decrease long-term treatment complications and improve the quality of life. As an important APBI modality, intraoperative radiotherapy (IORT) is introduced in BC treatment within a shorter time than other radiotherapeutic techniques. By using dedicated linear accelerators or novel mobile devices, it can directly deliver a single radiation dose to tumor bed in the operating room. IORT improves the accuracy of radiotherapeutic administration to protect normal tissues from damage. Some studies have shown that IORT can significantly improve the survival of BC patients administered with BCS and has a good cosmetic effect. A meta-analysis by Zhang et al. revealed that IORT has less side effects, better cosmetic effects, undifferentiated breast cancer and non-breast cancer mortality rates than those of WBRT. However, the risk of ipsilateral breast tumor recurrence was significantly higher with IORT than with WBRT. This meta-analysis included 4 studies, 2 of which were non-randomized control trials (RCTs), suggesting that this study was prone to biases, such as selection bias. Therefore, it is necessary for more meta-analyses to use high-quality studies when investigating the clinical efficacy and safety of IORT versus WBRT in early stage $B C$ patients receiving BCS based on available RCTs.

Condition being studied: Radiotherapeutic approaches have been developed to overcome the problems associated with WBRT. Among them, accelerated partial breast irradiation (APBI) is gradually becoming a surrogate to WBRT, because it can effectively shorten treatment time to 1-2 weeks, decrease long-term treatment complications and improve the quality of life. As an important APBI modality, intraoperative radiotherapy (IORT) is introduced in BC treatment within a shorter time than other radiotherapeutic techniques. By using dedicated linear accelerators or novel mobile devices, it can directly deliver a single radiation dose to tumor bed in the operating room. IORT improves the accuracy of radiotherapeutic administration to protect normal tissues from damage. Some studies have shown that IORT can significantly improve the survival of BC patients administered with BCS and has a good cosmetic effect.

\section{METHODS}

Search strategy: All eligible RCTs that compared IORT to WBRT in early stage BC patients were identified from PubMed, the Cochrane Library, Science Direct and China Biology Medicine databases up to October, 2019. The keywords used include: "breast neoplasms", "breast cancer", "breast carcinoma", "radiotherapy", "radiation", "intraoperative" and "IORT". We manually searched the reference lists of relevant reviews while abstracts from international conferences were also reviewed. Publication languages were limited to English and Chinese.

Participant or population: Eligible patients were to conform to the following criteria: $i$. Histologically confirmed as stage I or II BC patients who were suitable for BCS; ii. No preoperative anti-cancer treatments; iii. No other site cancer besides breast; iv. No serious organ (liver, kidney or heart et al) dysfunction and v. Randomly assigned to receive IORT or WBRT. The exclusion criteria were: i. Studies examining IORT as a "boost dose" followed by WBRT; ii. Tumor location was not easily accessible by the IORT equipment, such as in the tail of the breast; iii. Loss of follow-up rate that was higher than $20 \%$; iv. The ones with shorter follow-up for multiple articles presenting the same clinical trial and v. Non-RCTs. In this study, the IORT group was defined as patients receiving BCS and IORT while the WBRT group was defined as patients receiving $B C S$ followed by WBRT.

Intervention: IORT.

Comparator: WBRT.

Study designs to be included: RCT. 
Eligibility criteria: Eligible patients were to conform to the following criteria: $i$. Histologically confirmed as stage I or II BC patients who were suitable for BCS; ii. No preoperative anti-cancer treatments; iii. No other site cancer besides breast; iv. No serious organ (liver, kidney or heart et al) dysfunction and v. Randomly assigned to receive IORT or WBRT. The exclusion criteria were: i. Studies examining IORT as a "boost dose" followed by WBRT; ii. Tumor location was not easily accessible by the IORT equipment, such as in the tail of the breast; iii. Loss of follow-up rate that was higher than $20 \%$; iv. The ones with shorter follow-up for multiple articles presenting the same clinical trial and v. Non-RCTs. In this study, the IORT group was defined as patients receiving $B C S$ and IORT while the WBRT group was defined as patients receiving $B C S$ followed by WBRT.

Information sources: Two reviewers independently extracted detailed information regarding the publication year, first author, median follow-up time, radiotherapeutic planning, survival data and radiotherapeutic-associated adverse effects (AEs) from each trial. Discrepancies were resolved by discussions with a third author to reach a consensus. In our metaanalysis, recurrence or death within two years after adjuvant treatment was defined as short-term survival, while that with more than two years was defined as long-term survival.

Main outcome(s): 1) OS analysis for IORT versus WBRT; 2)DFS analysis for IORT versus WBRT.

Additional outcome(s): 1) Local recurrence analysis for IORT versus WBRT; 2)Radiotherapy-related AEs analysis for IORT versus WBRT.

Data management: Two reviewers independently extracted detailed information regarding the publication year, first author, median follow-up time, radiotherapeutic planning, survival data and radiotherapeutic-associated adverse effects (AEs) from each trial. Discrepancies were resolved by discussions with a third author to reach a consensus. In our metaanalysis, recurrence or death within two years after adjuvant treatment was defined as short-term survival, while that with more than two years was defined as long-term survival.

Quality assessment / Risk of bias analysis: We used the Newcastle-Ottawa Scale (NOS) to assess the quality of each included study. Scores $\geq 7$ were considered high quality. We used a "star system" for case-control studies.

Strategy of data synthesis: Quality of the enrolled RCTs was independently evaluated by two authors (Yuan-Yuan Chen and Min Sun) according to the Cochrane Collaboration's risk of bias tool, which includes the adequacy of random sequence generation, allocation concealment, blinding of participants and personnel, blinding of outcome assessment, incomplete outcome data, selective outcome reporting and other bias. Each item was assessed as low, high and unclear risk of bias. If there was any disagreement, a third author (Lin-Wei Wang) reviewed the materials again to reach a consensus.

Subgroup analysis: None.

Sensibility analysis: None.

Language: Searches were limited to English-language publications.

Country(ies) involved: China.

Other relevant information: None.

Keywords: Breast cancer; Intraoperative radiotherapy; Whole breast radiotherapy; Meta-analysis.

Contributions of each author:

Author 1 - Tian Li - Author 1 drafted the manuscript.

Email: fmmult@hotmail.com

Author 2 - Min Sun - The author participated in study design and performed data collection and data analysis. 